\title{
Fluid-containing emphysematous bullae: a spectrum of illness
}

\author{
D. Chandra*,\#, S.R. Rose ${ }^{\#}$, R.B. Carter ${ }^{\#, \mp}$, D.M. Musher ${ }^{\#,+}$ and R.J. Hamill ${ }^{\#,+}$
}

ABSTRACT: Fluid-containing emphysematous bullae are an under-reported complication of chronic obstructive pulmonary disease. The roles of bronchoscopy in the work-up and of antibiotics in the treatment are undefined.

This study reports the combined results from the analysis of 16 cases treated at the present authors' institution and $\mathbf{3 6}$ previously reported cases.

The median age at presentation was 58 yrs and the median duration of follow-up was 60 weeks. A third of the patients were asymptomatic, while two-thirds presented with symptoms, including $10 \%$ who had evidence of a severe lung infection. Methicillin-resistant Staphylococcus aureus, Pseudomonas aeruginosa and Bacteroides melaninogenicus were cultured from the bullae fluid in three symptomatic patients. Sputum and blood cultures were uninformative. Bronchoscopy, performed in two-thirds of the cases, added no diagnostic information. Antibiotic treatment did not result in a more rapid resolution of the air fluid level. Percutaneous drainage was safe and effective in four patients.

In conclusion, patients with fluid-containing bullae present with a spectrum of illness. Antibiotic treatment does not hasten radiographic resolution and bronchoscopy has no diagnostic or therapeutic role.

KEYWORDS: Chronic obstructive pulmonary disease, emphysema, fluid-containing emphysematous bulla, infected emphysematous bulla, lung bulla

luid-containing emphysematous bullae are an unusual complication of chronic obstructive pulmonary disease (COPD). As patients with COPD can develop a variety of cavitary lung lesions, identifying the correct diagnosis can be challenging [1-3]. The diagnosis of fluid-containing emphysematous bulla can only be made with certainty if there is interval development of a fluid level in a pre-existing emphysematous bulla [4,5]. Only a few of the small number of studies available have utilised this unequivocal criterion to establish the diagnosis in their patients [5-9]. The role of bronchoscopy in the work-up and of antibiotics and percutaneous drainage in treatment remains undefined. The present study reports the combined results from 16 cases of fluid-containing emphysematous bullae treated at the authors' institution and 36 previously reported cases.

\section{MATERIALS AND METHODS}

Identification of new cases

Due to the low incidence of fluid-containing emphysematous bullae a retrospective study design was chosen. As there is no International Classification of Diseases code for this diagnosis [10], a computerised search strategy was used to locate cases. Using the computerised medical records of the Michael E. DeBakey VA Medical Center (Houston, TX, USA), a search was carried out for radiology reports and discharge summaries containing the words "fluid level", "infected", "bulla" and "cyst" in varying combinations. In total, 353 documents were located dating from May 1991 to January 2007. These were then screened manually to select cases where there was unequivocal evidence of interval development of an airfluid level in a pre-existing emphysematous bulla. Thus, 18 cases were selected; two cases could not be included because essential parts of the medical record were not available. All patients had an extensive smoking history with clinical and spirometric evidence of obstructive airway disease.

\section{Identification of prior studies}

Using MEDLINE, all studies from 1966 to February 2007 were searched. Due to the lack of a medical subject heading for emphysematous bullae, a text search for "bulla", "emphysema", "cyst", "fluid" and "infection" in varying combinations was carried out. The reference lists of all identified manuscripts were also searched. In total, seven case series were found [3, 5-9, 11]; case reports containing individual cases were not
AFFILIATIONS

${ }^{*}$ Dept of Epidemiology, Harvard School of Public Health, Boston, MA,

"Depts of Medicine, and

"Paediatrics, Baylor College of Medicine, and

+Division of Infectious Diseases, Michael E. DeBakey VA Medical Center, Houston, TX, USA.

CORRESPONDENCE

D. Chandra

Dept of Epidemiology

Harvard School of Public Health

677 Huntington Ave Boston

MA

USA

Fax: 16179339345

E-mail: dchandra@hsph.harvard.edu

Received:

November 242007

Accepted after revision:

March 282008

STATEMENT OF INTEREST

None declared. 
included. The case series were reviewed by one of the authors (D. Chandra) to identify those where the fluid was noted to have developed in pre-existing emphysematous bullae. Four studies were selected adding a further 36 cases, which led to a final sample size of 52 cases [5-8].

Data on 34 variables was extracted from each of the 16 medical records and the four published articles. All statistical tests used were conventional. The study protocol, which included waiver of patient consent, was approved by the Institutional Review Board at Baylor College of Medicine (Houston).

\section{RESULTS}

Table 1 describes the demographics, clinical features at presentation and findings on initial work-up. Table 2 provides information on treatment and follow-up. Pulmonary examination did not reveal findings beyond those expected in patients with advanced COPD. Sputum cultures grew a variety of organisms generally implicated in respiratory infections in COPD patients; however, none could be confirmed as causative because bulla fluid culture results were not available in those patients, and blood cultures were uniformly negative.

\section{Microbiology of infected bullae}

Culture results from bullae fluid were available in four patients. The first patient was a 58-yr-old male with severe

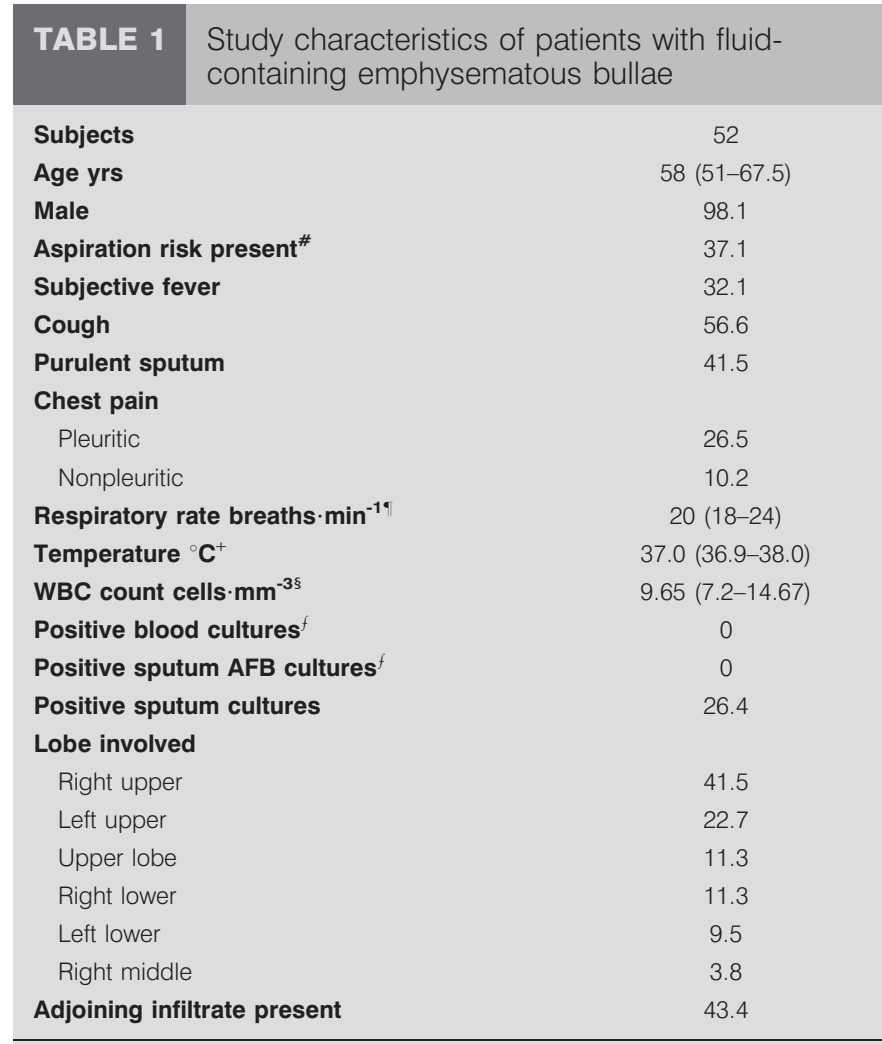

Data are presented as $\mathrm{n}$, median (interquartile range) or per cent. WBC: white blood cell; AFB: acid fact bacilli. "* available for 42 patients; ": available for 15 patients; ${ }^{+}$: available for 39 patients; ${ }^{\text {s. }}$ : available for 28 patients; ${ }^{f}$ : performed in 17 patients.
COPD (forced expiratory volume in one second $18 \%$ predicted), who was receiving oral clarithromycin and ethambutol for treatment of pulmonary Mycobacterium avium-intracellulare infection detected 6 months previously. The patient presented to the Michael E. DeBakey VA Medical Center (Houston) in 1996 with a 3-week history of fever and cough productive of green sputum. On admission the patient was breathing comfortably on room air, had a temperature of $36.9{ }^{\circ} \mathrm{C}$, a white blood cell (WBC) count of 17,000 cells $\cdot \mathrm{mm}^{-3}$ and a chest radiograph which showed interval development of an air-fluid level in a right upper lobe bulla. Percutaneous needle aspiration was performed 5 days after admission without placement of an indwelling catheter. Fluid cultures yielded Pseudomonas aeruginosa. The resolution of symptoms and disappearance of the air-fluid level was associated with 14 days of treatment with ceftazidime and gentamicin.

The second patient, a 57-yr-old male who presented in 2005, underwent drainage with an indwelling catheter with therapeutic intent on admission as he was extremely ill (temperature of $38.9{ }^{\circ} \mathrm{C}$ and WBC count of 34,700 cells $\cdot \mathrm{mm}^{-3}$ ). On entering the cavity, $500 \mathrm{~mL}$ of pus was aspirated and cultures grew Methicillin-resistant Staphylococcus aureus. There was dramatic improvement in symptoms after drainage. Further details of the patient's clinical course have been published previously [4].

The third and fourth patients have been reported previously by PETERs et al. [7]. One patient recovered uneventfully after drainage of the fluid containing bulla. Cultures grew Bacteroides melaninogenicus. However, culture results where negative for the fourth patient who had received empiric antibiotic treatment for 6 days prior to aspiration. Further details of the clinical presentation of these two patients have been published previously [7].

\section{Work-up, treatment and follow-up}

Bronchoscopy was performed in two-thirds of the patients but the results were noncontributory. As in the sputum cultures, bronchial washing yielded organisms that could not be confirmed as causative by bullae fluid cultures or blood cultures. Cytology for malignant cells was uniformly negative. Agents from almost every antibiotic class available were used

\begin{tabular}{l} 
TABLE $2 \begin{array}{l}\text { Bronchoscopy, treatment and follow-up for } 52 \\
\text { patients with fluid-containing emphysematous } \\
\text { bullae }\end{array}$ \\
$\begin{array}{l}\text { Antibiotic prescribed } \\
\text { Percutaneous aspiration performed }\end{array}$ \\
$\begin{array}{l}\text { Bronchoscopy performed } \\
\text { Time to radiographic resolution of } \\
\text { fluid level days }\end{array}$ \\
$\begin{array}{l}\text { Reduction or complete scarring of } \\
\text { affected bulla by end of follow-up }\end{array}$ \\
$\begin{array}{l}\text { Duration of follow-up days } \\
\text { (20.7-142.5) }\end{array}$ \\
\hline
\end{tabular}

Data are presented as per cent or median (interquartile range). ${ }^{*}$ : an indwelling catheter was left in place in half of the patients who underwent aspiration; $\because$ available for 47 patients. 
for treatment. Antibiotic use was not associated with time to resolution of the air-fluid level in patients with $(\mathrm{p}=0.81)$ or without symptoms $(p=0.52)$.

\section{DISCUSSION}

The pathogenesis of fluid accumulation in emphysematous bullae is controversial. The benign nature of this illness in most patients led MAHLER and co-workers $[6,12]$ to propose that the fluid was sterile and developed as a reaction to inflammation in the surrounding lung. This was clearly not the case in at least three out of the four patients who underwent bullae aspiration in the present study. However, all these patients had symptoms of a lung infection. The current authors believe that the hypothesis of MAHLER and co-workers [6, 12] may only hold true for patients who are asymptomatic, although it will remain unproven until bulla fluid from such patients is aspirated and analysed. In the absence of published data on the portal of entry of bacteria into the bulla, it can be speculated that the infection arises from the surrounding lung parenchyma or via haematogenous spread.

Patients with fluid-containing emphysematous bullae present with a spectrum of illness varying from an absence of symptoms to the presence of symptoms and a severe lung infection (i.e. temperature $\geqslant 38.3{ }^{\circ} \mathrm{C}$ or $\mathrm{WBC}$ count $\geqslant 15,000$ cells $\cdot \mathrm{mm}^{-3}$ ). A majority of patients, however, are symptomatic without a severe infection. The presence of this spectrum of illness has not been described previously. Contrary to prior reports [5], no correlation was found between the presence of symptoms of an infection and the presence of an infiltrate in the parenchyma surrounding the bulla $(\mathrm{p}=0.528)$.

Some authors have recommended bronchoscopy to rule out an occult malignancy or mycobacterial infection masquerading as fluid-containing bullae $[6,7]$. The results from the present study strongly argue against this recommendation.

Radiological evidence of the existence of a bulla prior to the accumulation of fluid is a sine qua non for the diagnosis of fluidcontaining bulla $[4,6]$. Its presence greatly simplifies the management of the COPD patient who presents with a new air-fluid level on a chest radiograph. When prior radiology studies are not available, the presence of other bullae in a patient with established obstructive airway disease, a thin-walled cavity and disproportionately less symptoms than the chest radiograph would all raise suspicion for this diagnosis [6, 7].

Medical management of fluid-containing bullae has been controversial because it is unclear if an antibiotic should be given, and, if so, which agent should be chosen [5-7]. The absence of an association between antibiotic treatment and time to resolution of the fluid level leaves little justification for routine antibiotic use in asymptomatic patients. The efficacy of antibiotics is harder to judge in symptomatic patients in the current study because no data was available on time to resolution of symptoms or fever, which are more meaningful markers of recovery than resolution of the air-fluid level. Confusion regarding the antibiotic of choice stems from uncertainty about the microbiological aetiology of these infections [5-7]. The only conclusion that can be drawn from the present microbiological results is that causative organisms can vary widely and if a rapid clinical response with empiric antibiotics does not occur then obtaining bulla fluid cultures may be prudent.

Percutaneous drainage of fluid-containing lung bullae has previously been strongly discouraged [5-7]. The four patients who underwent this procedure all experienced rapid improvement in symptoms without procedural complications.

The current study is limited by its retrospective design. Wide variation in management probably existed between treating physicians. However, given the very low incidence of the condition, this was the only type of study design feasible. Although the strict inclusion criteria resulted in a smaller sample size, it was felt that the inclusion criteria were necessary to ensure the quality of the data.

In conclusion, patients with fluid-containing bullae can present with a severe lung infection. Data on causative organisms is limited, and includes a wide spectrum of bacteria. Based on this limited evidence, the present authors suggest that management decisions be tailored to the acuity of presentation. For asymptomatic patients antibiotic treatment does not appear beneficial. Symptomatic patients may benefit from antibiotic treatment, while those with severe symptoms, high-grade temperature $\left(\geqslant 38.3^{\circ} \mathrm{C}\right)$ and leukocytosis may benefit from additional percutaneous drainage. Bronchoscopy should not be performed unless there is clear indication of an underlying malignancy, such as a mass adjoining the affected bulla.

\section{ACKNOWLEDGEMENTS}

The authors appreciate the efforts of G.O. Welch (computer specialist; Dept of Information Management, Michael E. DeBakey VA Medical Center, Houston, TX, USA) for running the computerised search protocol for this study.

\section{REFERENCES}

1 Rothstein E. Infected emphysematous bullae: report of five cases. Am Rev Tuberc 1954; 69: 287-296.

2 Rothstein E, Harley BF Jr. Fluid levels in emphysematous bullae. Dis Chest 1962; 42: 620-625.

3 Sanford HS, Green RA. Air-fluid levels in emphysematous bullae. Dis Chest 1963; 43: 193-199.

4 Chandra D, Soubra SH, Musher DM. A 57-year-old man with a fluid-containing lung cavity: infection of an emphysematous bulla with methicillin-resistant Staphylococcus aureus. Chest 2006; 130: 1942-1946.

5 Leatherman JW, McDonald FM, Niewohner DE. Fluidcontaining bullae in the lung. South Med J 1985; 78: 708-710.

6 Mahler DA, Gerstenhaber BJ, D’Esopo ND. Air-fluid levels within lung bullae associated with pneumonitis. Lung 1981; 159: 163-171.

7 Peters JI, Kubitschek KR, Gotlieb MS, Awe RJ. Lung bullae with air-fluid levels. Am J Med 1987; 82: 759-763.

8 Rubin EH, Buchberg AS. Capricious behavior of pulmonary bullae developing fluid. Dis Chest 1968; 54: 546-549.

9 Wahbi ZK, Arnold AG. Spontaneous closure of a large emphysematous bulla. Respir Med 1995; 89: 377-379.

10 CDC WONDER International classification of diseases. http://wonder.cdc.gov/wonder/cgi-bin/asp/ICDFinder.asp Date last accessed: January 20, 2008. Date last updated: August 29, 2007. 
11 Douglas AC, Grant IW. Spontaneous closure of large pulmonary bullae: a report on three cases. Br J Tuberc Dis Chest 1957; 51: 335-338.
12 Mahler DA, D'Esopo ND. Peri-emphysematous lung infection. Clin Chest Med 1981; 51-57. 